\title{
Implications of endogenous roles of transporters for drug discovery: hitchhiking and metabolite-likeness
}

\section{Douglas B. Kell}

In a recent article (Nat. Rev. Drug Discov. 14, 29-44; 2015) ${ }^{1}$, Nigam asks the question "what do drug transporters really do?", and discusses the evidence for endogenous roles of transporters in the ATP-binding cassette (ABC) and solute carrier (SLC) transporter families, which have endogenous substrates that include metabolites, antioxidants, signalling molecules, hormones, nutrients and neurotransmitters. Although the article did not focus on the role of transporters in drug uptake, I thought that it could be valuable to highlight some implications for drug discovery of the increasing knowledge on the endogenous substrates and functions of the $>500 \mathrm{SLCs}^{2}$ and $\sim 1,000$ transporters encoded overall in the human genome.

In particular, as we have noted in multiple articles - beginning with our article in this journal in 2008 (REF. 3) — "drugs may 'hitchhike' on carriers or transporters that act on natural endogenous substrates (albeit that these substrates or carriers are often unknown)". Since then $^{3}$, the available evidence for the roles of endogenous transporters in transporting drugs has strengthened considerably ${ }^{2}$, adding substantial support for the hypothesis that not only do drugs use endogenous transporters widely, but also that any diffusion through the phospholipid bilayer of cell membranes is normally negligible (see REF. 4 for an updated review). Winter et al. ${ }^{5}$ provide an excellent example of this: genetic removal of just a single transporter decreases by several-100-fold the uptake and toxicity of the candidate anticancer drug YM155 - that is, to less than 1\% of their original values.

As many drugs need to cross at least one cellular membrane to exert their pharmacological effects, this hypothesis has important implications for drug discovery strategies. Chemical starting points for novel small-molecule drugs are typically discovered by two kinds of strat$\mathrm{egy}^{6}$. The first (which is more classical, although it is now in the midst of a resurgence) is function-first, phenotypic screening, in which the modes of action of screening 'hits' are rarely known in advance, albeit that these active compounds must bind to substances involved in biochemical processes. This typically involves initial screening in cellular or animal models, and thus active compounds derived from such screens will generally have some ability to cross cellular membranes (although membrane permeability is just one of the multiple factors that affect bioavailability in vivo).

The second, more recent strategy involves molecular methods that are based on hypotheses about the role of a particular target in disease, often coupled with structural modelling of a binding site on that target. In this case, initial (typically high-throughput) screens often involve assay formats that do not require cellular permeability for activity. However, for a candidate drug, some cellular permeability is generally crucial, and substantial work by medicinal chemists to optimize hit compounds from these screens may be needed to achieve this. This has fuelled the development of many assays and computational approaches that attempt to predict the ability of compounds (particularly those in screening libraries) to cross biological membranes and achieve sufficient bioavailability in vivo, not always successfully.

So, how does this relate to the growing knowledge of the endogenous biological roles of transporters, such as those transporting metabolites? First, it is important to note that both compound-screening strategies have often used endogenous molecules as the starting point for the search for active compounds. For phenotypic screening strategies, particularly historically, the starting point has frequently been either an endogenous molecule (or something closely related) that had a biological activity linked to the desired pharmacological effect - for example, antimetabolites as anticancer drugs, or adrenaline-related compounds as drugs for vascular disorders. In the case of target-oriented drug discovery strategies, the binding site on the target has often been one for an endogenous molecule, such as the ATP-binding site on protein kinases.

Given this, it would not necessarily be surprising that successful (that is, marketed) drugs are more like endogenous molecules than are the typical structures found in drug discovery screening libraries - a characteristic that has been termed 'metabolite-likeness' (REF. 7). This is indeed found to be the case ${ }^{7}$, and actually many drugs do have the same target binding site as a human metabolite (as noted in REF. 7). However, whereas the likelihood of molecular similarity between compounds that have similar biological effects that are mediated by interactions with the same biological targets may be widely appreciated, another aspect of the potential importance of metabolite-likeness appears to be less so. That is, compounds that are more similar to endogenous metabolites may be more likely to be able to hitchhike on the transporters for those metabolites and thereby reach their pharmacological targets in vivo. Importantly, this does not imply that a compound necessarily acts pharmacologically on the endogenous targets of the metabolites to which it is similar; rather, it just needs to be sufficiently similar to one or more metabolites to be able to exploit the same transporters to cross cellular membranes (and some of these transporters may recognize a wide range of substrates). As a related aside, the possibility that 'natural-product-likeness' may be a valuable property in screening libraries has been discussed extensively (for example, see REF. 8). Following a similar rationale, the value of screening natural-product-like compounds may be in part because natural products have evolved both to interact with their pharmacological targets and to hitchhike on transporters to reach these targets.

So, there could be substantial benefits for drug screening from greater acknowledgement of the importance of 'metabolite-likeness' as well as from increasing our knowledge of the substrate specificities and distribution of transporters $^{3,8}$. Filters could be applied to screening libraries (as is widely done at present with simple physicochemical filters such as the 'Rule of 5') to increase the likelihood that screening hits have sufficient cellular permeability ${ }^{8}$. Such filters could become more sophisticated as the knowledge of which transporters are involved in transporting which substrates (and the relevant metabolic networks ${ }^{6}$ ) increases - perhaps even allowing particular tissues to be targeted more specifically through tailoring of drug properties; for example, to exploit tissuespecific expression of the relevant transporters on which the drugs are hitchhiking ${ }^{3,4}$.

To date, the expression levels of only a relatively small number of the better-known drug transporters have been manipulated. However, the newly acquired abilities to manipulate transporter expression levels systematically and in a high-throughput manner, whether by genome editing or other means ${ }^{2,4,5}$, will no doubt allow us to make considerable progress in answering the question of which transporters are used by specific drugs. Coupling this with progress in the understanding of the endogenous biological roles of transporters 


\section{CORRESPONDENCE}

could have a key role in identifying drug candidates that have the ability to cross biological membranes at the relatively modest rates necessary ${ }^{9}$ to exert the desired pharmacological effect at the desired location, using principles based on those of natural selection ${ }^{10}$.

Douglas B. Kell is at the School of Chemistry, The Manchester Institute of Biotechnology, and the Centre for Synthetic Biology of Fine and Speciality Chemicals (SYNBIOCHEM). The University of Manchester, 131 Princess Street, Manchester M1 7DN, UK. dbk@manchester.ac.uk

doi:10.1038/nrd.2015.44 Published online 3 Feb 2016
1. Nigam, S. K. What do drug transporters really do? Nat. Rev. Drug Discov. 14, 29-44 (2015).

2. César-Razquin, A. et al. A call for systematic research on solute carriers. Cell 162, 478-487 (2015).

3. Dobson, P. D. \& Kell, D. B. Carrier-mediated cellular uptake of pharmaceutical drugs: an exception or the rule? Nat. Rev. Drug Discov. 7, 205-220 (2008)

4. Kell, D. B. ¿ Oliver, S. G. How drugs get into cells: tested and testable predictions to help discriminate between transporter-mediated uptake and lipoidal bilayer diffusion. Front. Pharmacol. 5 231 (2014).

5. Winter, G. E. et al. The solute carrier SLC35F2 enables YM155-mediated DNA damage toxicity. Nat. Chem. Biol. 10, 768-773 (2014).

6. Kell, D. B. Finding novel pharmaceuticals in the systems biology era using multiple effective drug targets, phenotypic screening, and knowledge of transporters: where drug discovery went wrong and how to fix it. FEBS J. 280, 5957-5980 (2013).
7. O'Hagan, S., Swainston, N., Handl, J. \& Kell, D. B. A 'rule of 0.5 ' for the metabolite-likeness of approved pharmaceutical drugs. Metabolomics 11, 323-339 (2015).

8. Jayaseelan, K. V., Moreno, P. Truszkowski, A., Ertl, P. \& Steinbeck, C. Natural product-likeness score revisited: an open-source, open-data implementation. BMC Bioinformatics 13, 106 (2012).

9. O'Hagan, S. \& Kell, D. B. The apparent permeabilities of Caco-2 cells to marketed drugs: magnitude, and independence from both biophysical properties and endogenite similarities. PeerJ. 3 , e1405 (2015).

10. Mendes, P., Oliver, S. G. \& Kell, D. B. Fitting transporter activities to cellular drug concentrations and fluxes: why the bumblebee can fly. Trends Pharmacol. Sci. 36, 710-723 (2015).

Competing interests statement

The author declares no competing interests. 